\title{
Optical coherence tomography in primary percutaneous coronary intervention: Helping to clear the angiographic fog
}

\author{
Petru Mester, Mihaela Rata, Lionel Mangin, Loic Belle \\ Interventional Cardiology Department, Centre Hospitalier de la Region d'Annecy, France
}

Correspondence: Loic Belle. Address: Interventional Cardiology Department, Centre Hospitalier de la Region d'Annecy, France. Email: loic.belle@wanadoo.fr

Received: January 18, 2016

Accepted: March 14, 2016

Online Published: March 22, 2016

DOI : $10.5430 /$ ijdi.v3n2p1

URL: http://dx.doi.org/10.5430/ijdi.v3n2p1

\begin{abstract}
The finding of patent arteries on coronary angiography in patients hospitalized for ST-segment elevation myocardial infarction (STEMI) is not a rare phenomenon in daily practice. However, in a subgroup of these cases, namely patients with a history of drug-eluting stent implantation, intracoronary optical coherence tomography (OCT) may reveal angiographically silent stent thrombosis. We describe the case of a 64-year-old man admitted with clinical, electrocardiographic and biological features of STEMI, despite an angiographically patent stent and non-significant lesions in the coronary arteries. We emphasize the role of OCT in diagnosing the culprit artery, the etiopathologic substrate and therapeutic management in such cases.
\end{abstract}

\section{Keywords}

Primary percutaneous coronary intervention, Very late stent thrombosis, Optical coherence tomography

\section{I ntroduction}

By inhibiting neointimal proliferation, the first-generation drug-eluting stents (DES) have substantially reduced the rate of in-stent restenosis, compared with bare-metal stents (BMS) ${ }^{[1]}$. However, the initial enthusiastic success was overshadowed by evidence of an increased risk of late and very late stent thrombosis (VLST), complications that are not typically encountered with BMS ${ }^{[2]}$. The key pathological mechanism for late stent thrombosis (occurring 1-12 months after implantation) is likely to be delayed arterial healing characterized by uncovered stent struts. Beyond this period, however, thorough investigations have revealed additional mechanisms of stent thrombosis such as localized hypersensitivity leading to positive vessel wall remodeling (encountered more frequently with sirolimus-eluting stents), excessive fibrin deposits with or without malapposition of stent struts (encountered more frequently with paclitaxel-eluting stents) and neoatherosclerosis ${ }^{[3]}$.

Optical coherence tomography (OCT) is an intracoronary high-resolution imaging technique. Axial resolution with OCT is 10-20 microns, being superior to intravascular ultrasound (100-200 microns) and other non-invasive coronary imaging techniques such as computed tomography coronary angiography and cardiac magnetic resonance. Among the applications, 
the high resolution of this imaging technique provides a detailed evaluation of the coronary wall. Therefore, OCT is a validated and useful imaging technique, especially in cases of angiographically unclear findings ${ }^{[4]}$.

We report a case of VLST (occurring 4 years after the index event) secondary to a rare cause of stent thrombosis, and the key role played by OCT in the diagnosis (of both stent thrombosis and the etiopathologic substrate) and in guiding therapeutic management.

\section{Case presentation}

We present the case of a 64-year-old man with a history of non-ST-segment elevation myocardial infarction and angiographic evidence of $90 \%$ stenosis of the distal left main coronary artery (LMCA) and the proximal left anterior descending artery (LAD), treated by DES implantation (CYPHER ${ }^{\circledR} 18 \mathrm{~mm} \times 3.5 \mathrm{~mm}$; Cordis) with a proximal optimization technique (Maverick ${ }^{\mathrm{TM}} 12 \mathrm{~mm} \times 4 \mathrm{~mm}$ balloon for distal left main coronary artery; Boston Scientific) in April 2011 (see Figure 1).
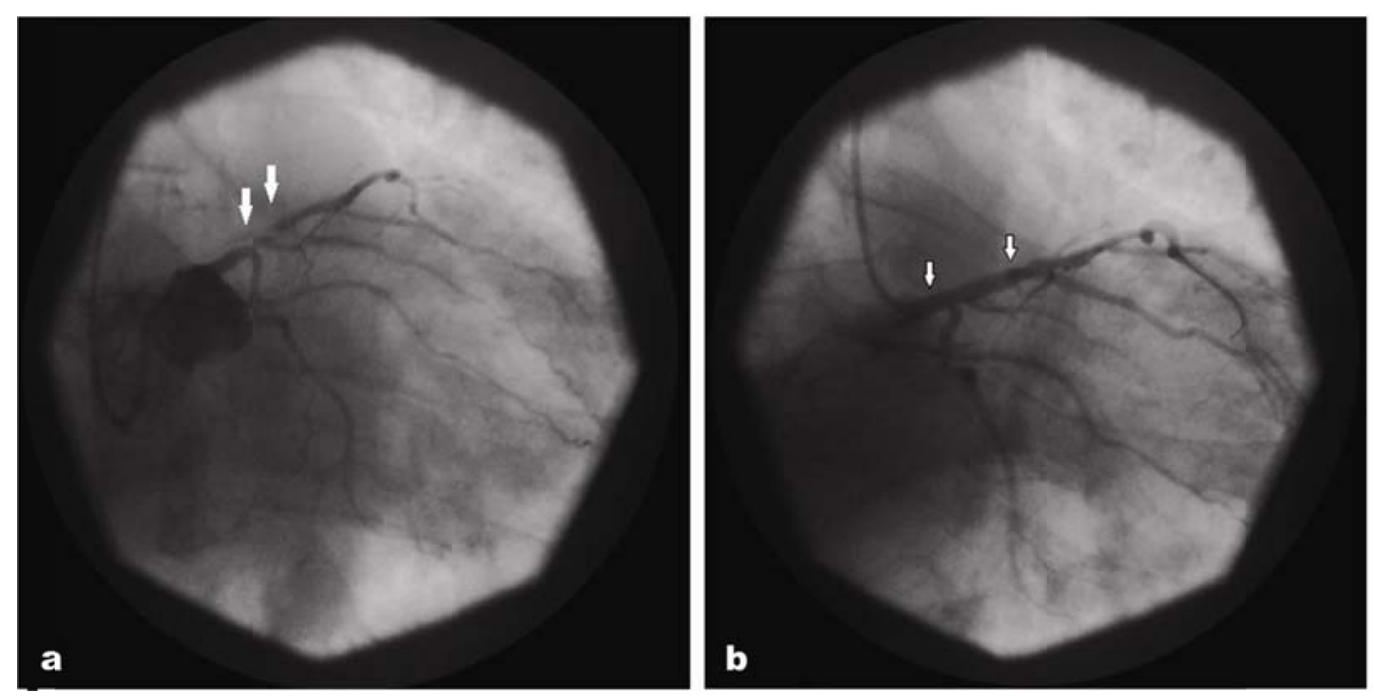

Figure 1. a. Coronary angiography, right anterior oblique caudal view, showing stenoses in the distal left main coronary artery and the proximal left anterior descending artery (white arrows); b. Coronary angiography, right anterior oblique caudal view, after stenting: distal left main coronary artery, proximal left anterior descending artery (white arrows)

The patient was admitted 4 years after the first coronary event (February 2015) with clinical, biological and electrocardiographic features of anterior ST-segment elevation myocardial infarction (STEMI), less than 12 hours after symptom onset.

We performed emergency coronary angiography 5 hours after symptom onset, which revealed a normal right coronary artery, a patent left coronary artery with a heterogeneous intrastent appearance in the distal LMCA and proximal LAD with contrast staining at this level and no visible thrombus (see Figure 2).

Left ventriculography highlighted apical akinesis. Before confirming the diagnosis of myocardial infarction without significant artery stenosis, we performed an OCT examination which revealed peri-stent pseudoaneurysmal vessel wall remodeling: an outward bulge in the luminal vessel contour between apposed stent struts, with a maximum depth of the bulge exceeding by far the actual strut thickness. The total length of the vessel wall remodeling process was calculated at $15 \mathrm{~mm}$ with an outward bulge depth between $0.5 \mathrm{~mm}$ and $0.9 \mathrm{~mm}$ (14\%-25\% of stent nominal diameter), with the findings corresponding to major evaginations ${ }^{[5]}$. The maximum diameter of the positive remodeled segment was evaluated at $\sim 6$ $\mathrm{mm}$ (see Figure 3). OCT also revealed a low burden of parietal thrombus at the distal part of the stent (see Figure 4). 
Figure 2. Coronary angiography, right anterior oblique caudal view, showing peri-stent staining (white arrows)
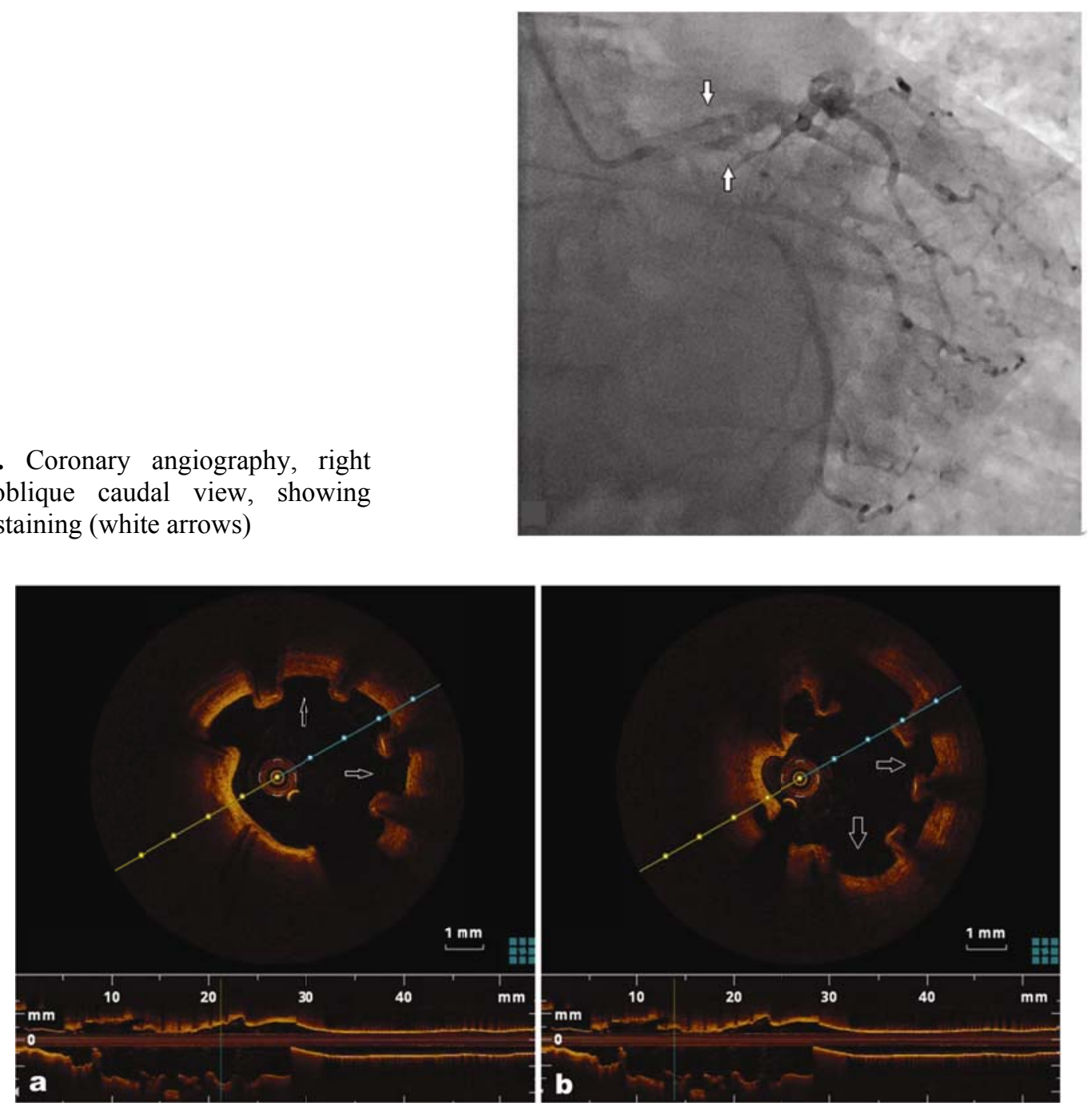

Figure 3. a. Optical coherence tomography image. Transverse axis shows peri-stent pseudoaneurysmal vessel wall remodeling (white arrows), with intra-stent diameter of $4.9 \mathrm{~mm}$ and intra-stent area of $17 \mathrm{~mm}^{2}$; the depth of evaginations varies between $14 \%$ and $25 \%$ of stent nominal diameter; the discrete intimal hyperplasia and the absence of positive remodeling (or evagination) between 6 and 10 hours may lead to an interpretation of plaque protrusion. Longitudinal axis shows positive remodeled peri-stent vessel wall process with a length of $15 \mathrm{~mm}$; b. Optical coherence tomography image. Transverse axis shows peri-stent pseudoaneurysmal vessel wall remodeling (white arrows), with the depth of evaginations varying between $14 \%$ and $25 \%$ of the stent nominal diameter; structure between 7 hours and 10 hours interpreted as thrombus with distal shadow cone. Longitudinal axis shows positive remodeled peri-stent vessel wall process with a length of $15 \mathrm{~mm}$

We therefore confirmed our diagnosis as anterior STEMI (peak troponin value was $8 \mu \mathrm{g} / \mathrm{L}$ with normal values $\leq 0.26 \mu \mathrm{g} / \mathrm{L}$ ) with thrombus formation secondary to positive peri-stent vessel remodeling.

The patient received optimal pharmacological treatment comprising aspirin $75 \mathrm{mg}$ daily, ticagrelor $180 \mathrm{mg}$ daily, low-molecular-weight heparin (enoxaparin administered initially as a $30 \mathrm{mg}$ intravenous bolus followed 15 minutes later by $1 \mathrm{mg} / \mathrm{kg}$ every $12 \mathrm{~h}$ ) and a glycoprotein IIb/IIIa inhibitor (tirofiban administered initially as a $25 \mu / \mathrm{kg}$ bolus followed by a maintenance infusion of $0.15 \mu / \mathrm{kg} / \mathrm{min}$ for 2 days). 
Two weeks later we performed a new OCT procedure that showed the disappearance of the thrombus, with persistent pseudoaneurysm in the vessel wall. We finally treated the lesion by balloon angioplasty (intrastent dilatation with $4,4.5$ and $5 \mathrm{~mm}$ balloons), obtaining a satisfactory result (see Figure 5).

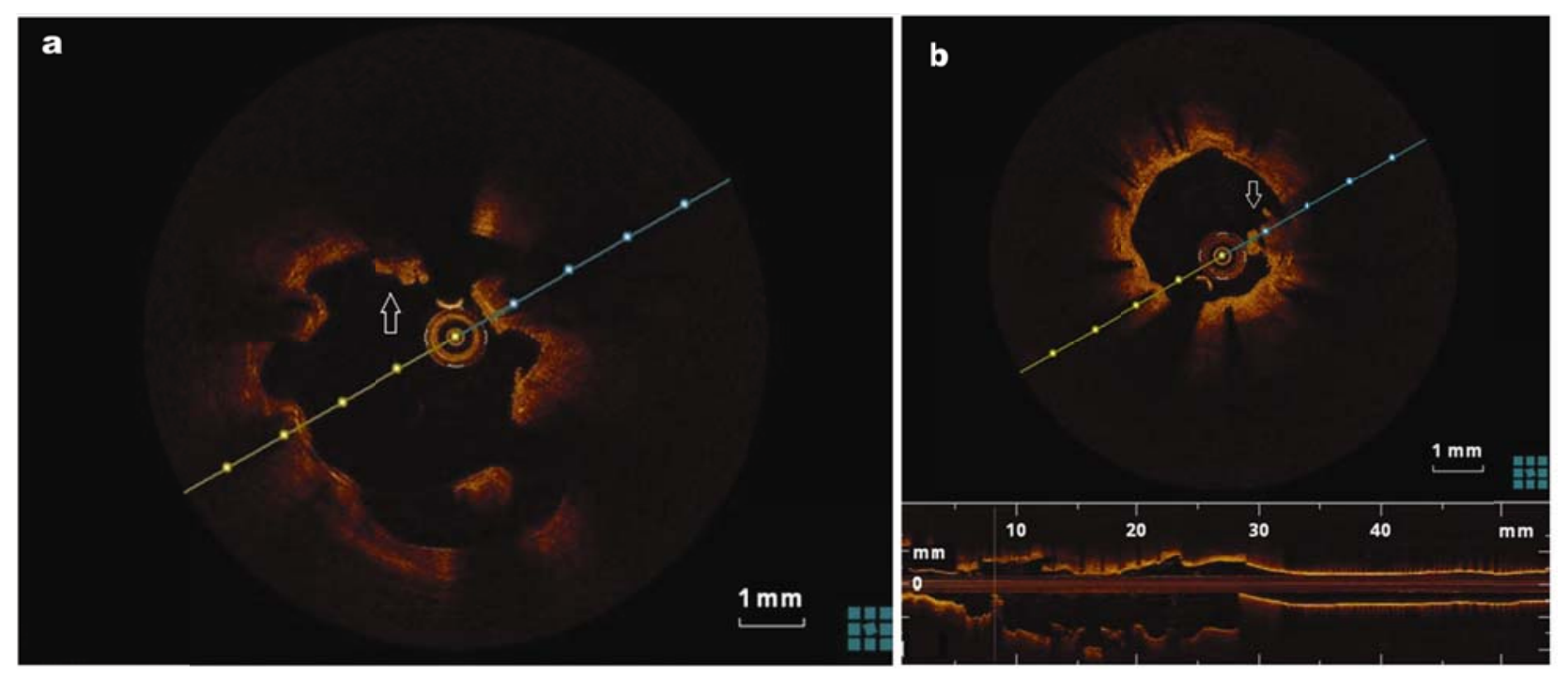

Figure 4. a. Optical coherence tomography image showing thrombus (white arrows) in the coronary segment, with vessel wall remodeling; b. Optical coherence tomography image showing thrombus (white arrows) at the level of the distal segment of the stent

Figure 5. Optical coherence tomography image showing the final result after intrastent balloon dilatation; microfissures are visible around 6 hours and 8 hours

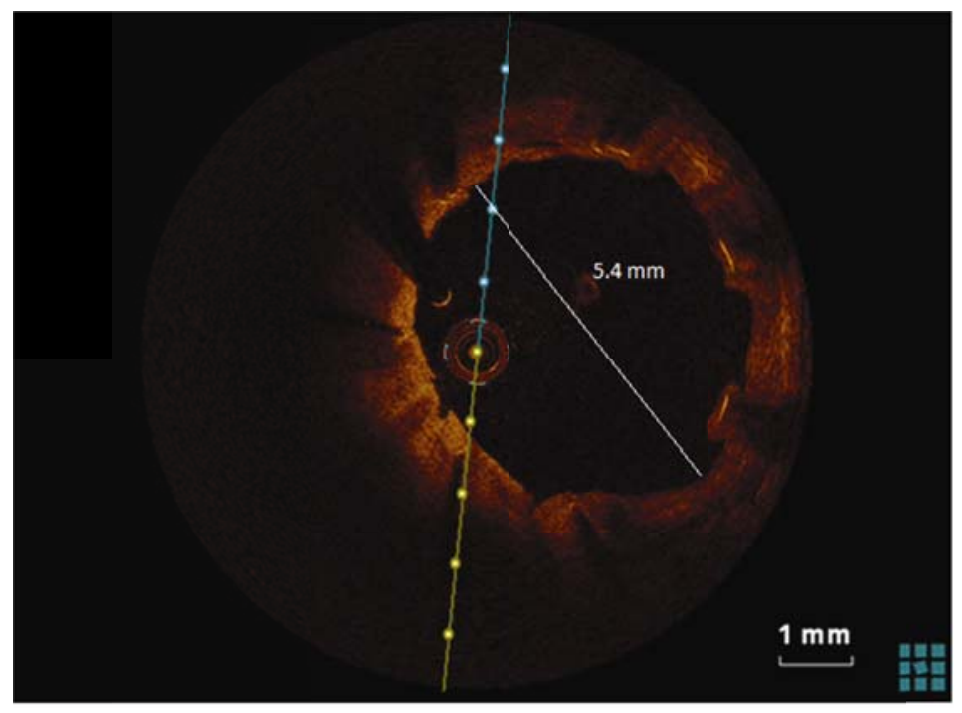

We discharged the patient 2 days later with a long-term dual antiplatelet treatment strategy.

\section{Discussion}

Here we describe the case of a man admitted with clinical, electrocardiographic and biological features of STEMI, despite an angiographically patent stent and non-significant lesions in coronary arteries - a phenomenon reported in daily practice with a frequency of $1 \%-12 \%{ }^{[6]}$. Nevertheless, published data support the fact that in patients with a history of stent implantation presenting with acute coronary syndromes, OCT may reveal angiographically silent stent thrombosis ${ }^{[7]}$. 
Similarly, in our case, initial angiography revealed non-obstructive coronary disease without a clear relation to the STEMI. The OCT revealed intrastent low burden thrombosis, thereby providing not only the culprit vessel, but also the probable morphopathologic basis for the acute coronary syndrome (thrombus).

In our case, coronary angiography showed a particular feature of abnormal contrast staining around the implanted stent, and an irregular vessel wall contour in that specific region. Imai and colleagues described a similar abnormal angiographic feature and named it peri-stent staining (PSS). This finding was defined as contrast extending peri-stent to $>20 \%$ of the stent diameter. These authors also observed that PSS in patients with sirolimus-eluting stent (SES) implantation was associated with higher rates of VLST at 3-year follow-up (compared with patients without PSS) ${ }^{[8]}$.

In addition, Mostafa and colleagues reported a series of 10 consecutive symptomatic patients after implantation of first-generation SESs, who had the same angiographic feature (peri-stent staining). The authors concluded, after intravascular ultrasound assessment, that PSS indicates possible aneurysm formation in the context of positive vessel wall remodeling ${ }^{[9]}$.

In light of this information, we performed a coronary OCT examination, which showed the definite feature of aneurysm formation secondary to positive remodeling. So, although it was angiographically suspected, it was the OCT that confirmed the underlying mechanism of stent thrombosis (positive vessel wall remodeling). Therefore, at least in our case, it seems that coronary angiography alone is not sufficient to clearly show the anatomical features in symptomatic patients after DES implantation. Symptoms resembling myocardial ischemia in these patients should not be analyzed superficially, and OCT should be performed for a better understanding of angiographic features.

Regarding the treatment of arterial vessel wall remodeling following DES implantation, there are no well-established strategies. Published articles describe various treatment options: stent implantation (DES as well as bare-metal stents); balloon angioplasty; or conservative treatment ${ }^{[9]}$. Even if we do not have solid scientific evidence for it, life-long dual antiplatelet therapy is a reasonable therapeutic option for these patients ${ }^{[8]}$. In our case, OCT was the tool that helped us to decide on an optimal treatment strategy (intrastent $5 \mathrm{~mm}$ balloon angioplasty) and to evaluate the final intracoronary result.

In conclusion, angiography interpretation may be ambiguous and OCT can be crucial for the detection of low burden VLST secondary to positive vessel wall remodeling in symptomatic patients with a history of DES implantation, and for the management of interventional treatment in this particular context.

\section{References}

[1] Daemen J, Serruys PW. Drug-eluting stent update 2007: part I. A survey of current and future generation drug-eluting stents: meaningful advances or more of the same? Circulation. 2007; 116(3): 316-328. PMid:17638940 http://dx.doi.org/10.1161/CIRCULATIONAHA.106.621342

[2] Camenzind E, Steg PG, Wijns W. Stent thrombosis late after implantation of first-generation drug-eluting stents: a cause for concern. Circulation. 2007; 115: 1440-55. PMid:17344324 http://dx.doi.org/10.1161/CIRCULATIONAHA.106.666800

[3] Nakazawa G. Stent thrombosis of drug eluting stent: pathological perspective. J Cardiol. 2011; 58(2): 84-91. PMid:21839616 http://dx.doi.org/10.1016/j.jjcc.2011.07.004

[4] Optical coherence tomography for coronary imaging. An article from the E-Journal of the ESC Council for Cardiology Practice. 2010; 9(12-13).

[5] Radu MD, Räber L, Kalesan B, et al. Coronary evaginations are associated with positive vessel remodelling and are nearly absent following implantation of newer-generation drug-eluting stents: an optical coherence tomography and intravascular ultrasound study. Eur Heart J. 2014; 35: 795-807. PMid:24132187 http://dx.doi.org/10.1093/eurheartj/eht344

[6] Kardasz I, De Caterina R. Myocardial infarction with normal coronary arteries: A conundrum with multiple aetiologies and variable prognosis: An update. Journal of Internal Medicine. 2007; 261: 330-348. PMid:17391108 http://dx.doi.org/10.1111/j.1365-2796.2007.01788.x

Published by Sciedu Press 
[7] Steigen SE, Holm NR, Butt N, et al. Clinical use of optical coherence tomography to identify angiographic silent stent thrombosis. Scand Cardiovasc J. 2014; 48(3): 156-160. PMid:24601754 http://dx.doi.org/10.3109/14017431.2014.900185

[8] Imai M, Kadota K, Goto T, et al. Incidence, risk factors, and clinical sequelae of angiographic peri-stent contrast staining after sirolimus-eluting stent implantation. Circulation. 2011; 123(21): 2382-2391. PMid:21576652

http://dx.doi.org/10.1161/CIRCULATIONAHA.110.003459

[9] Mostafa AE, Richardt G, Abdel-Wahab M. Symptomatic abnormal vessel wall reaction after implantation of the first generation sirolimus-eluting stent: a case series. Cardiovasc Revasc Med. 2012; 13(3): 196-200. PMid:22652316

http://dx.doi.org/10.1016/j.carrev.2012.02.008 